\title{
Obesity and pain sensitivity: Why should we care?
}

\section{Rebecca Price}

\section{McGill University}

The global rise in the prevalence of obesity has, perhaps not surprisingly, led to an increase in the number of surgical procedures performed in the morbidly obese. Gastric bypass surgery in particular has steadily increased in frequency since the early 1990s, reaching a plateau in 2004 with an average of 113,000 surgeries performed per year in the U.S. ${ }^{1}$ Respiratory depression due to perioperativelyadministered opioids is a potential serious complication of this surgery for the morbidly obese patient. ${ }^{2}$ Obesity is associated with concomitant respiratory dysfunction, particularly obstructive sleep apnea, ${ }^{2}$ placing obese patients at a higher risk for opioid-induced respiratory depression than non-obese patients.

Interestingly, previous literature suggests that obese persons demonstrate decreased pain sensitivity. ${ }^{3,45}$ However, this association is far from conclusive, as increased pain sensitivity ${ }^{6}$ has also been observed. Nevertheless, if true, decreased doses of opioid analgesics may be sufficient to treat post-surgical pain in obese patients and so reduce their risk of postoperative respiratory depression. Moreover, the mechanism by which pain sensitivity is decreased in obesity could provide new insights into pain perception relevant for both obese and non-obese individuals.

Still, it is difficult to draw definitive conclusions regarding the role of pain sensitivity in obesity due to the methodological flaws of previous studies. For instance, only study samples with wide age ranges (where age was not controlled) 3,4,5,6 and composed predominantly of female volunteers ${ }^{3,4}$ have been examined - despite the fact that both age and gender influence pain perception. In addition, previous studies did not account for the amount of local adiposity at sites used for pain testing (i.e. forearm). ${ }^{3,4,5,6}$ As such, any differences in pain sensitivity observed between obese and normal weight participants could be due to local factors affected by adiposity (e.g., the local chemical environment) rather than central, systemic contributions, minimizing the ability to draw conclusions regarding generalized alterations of pain sensitivity. Other methodological weaknesses of previous studies include pain tests that were poorly described ${ }^{5}$ or that lacked operational definitions of pain outcome measures. ${ }^{3,4,6}$ In pain studies that rely on subjective pain measurements, it is crucial that study subjects be given specific standardized instructions to avoid inconsistencies in pain assessment that may lead to invalid findings.

The true relationship between obesity and pain sensitivity, therefore, remains unclear. Future rigorous studies that address the limitations of the current literature are needed to definitively conclude if pain sensitivity is altered in obesity. Despite this uncertainty, I propose that obesity is more likely to be associated with decreased pain sensitivity due to underlying relationships with reward processing 7,8 and inflammation. ${ }^{9}$ So how could these relationships decrease pain sensitivity in obesity and why should we care?

Obesity may be the result of an addictive disorder in which patients are addicted not to drugs or alcohol, but to food. Much recent literature suggests that pain and reward are but two ends of the same spectrum, linked by neurotransmitters that have rewarding, as well as analgesic properties, including opioids8. Opioid agonists, such as morphine, are well-known effective modulators of pain, as well as appetite stimulants. ${ }^{3,4,6}$ Obesity is already known to be associated with increased levels of beta-endorphin ${ }^{\text {e.s.7 }}$, an endogenous opioid agonist. This suggests that obese individuals may be chronically rewarded, shifted toward the hedonic and away from the pain end of the spectrum and leading to overeating, as well as decreased pain perception.

Obesity is also a state of chronic inflammation in which cytokines, both pro-inflammatory cytokines, which increase pain sensitivity, as well as anti-inflammatory cytokines, are secreted directly from the adipose tissue and are systemically 
elevated compared to normal weight individuals. ${ }^{9}$ Moreover, it appears that secretion of anti-inflammatory markers may be greater than pro-inflammatory cytokines in obesity as is the case of interleukin-1beta and its proinflammatory counterpart interleukin-1. ${ }^{9}$ Recent literature in the pain field, particularly in inflammatory disorders, suggests it may be the ratio of pro- to anti-inflammatory markers that is clinically important. ${ }^{10}$ Hence, a decreased ratio may underlie decreased pain sensitivity in obesity.

Certainly, there may be other potential explanations for decreased pain sensitivity in obesity - perhaps related to excess adiposity and/or chronic overeating. Should future studies indeed demonstrate the presence of decreased pain sensitivity in obesity, we would have a unique human model for research that would allow us to answer clinically relevant questions, including investigating the interaction between pain and reward. Understanding decreased pain sensitivity in obesity also has the potential to uncover novel pharmaceutical targets for pain management. While we already know obesity is associated with elevated levels of a class of molecules that are the most effective analgesics currently available, perhaps other mechanisms or molecules contribute to decreased pain sensitivity in obesity. This could provide new avenues for pharmaceutical research, as well as a basis for safer dosing procedures of post-operative analgesics in the morbidly obese.

\section{Acknowlegments}

Dr. Petra Schweinhardt and Dr. Steven Backman for their support and supervision.

\section{References}

1. Livingston EH. The incidence of bariatric surgery has plateaued in the U.S. Am J Surg. 2010 Sept; 200(3):378-385.

2. Adams JP, Murphy PG. Obesity in anaesthesia and intensive care. $\mathrm{Br} \mathrm{J}$ Anaesth. 2000 July; 85(1):91-108.

3. Zahorska-Markiewicz B, Kucio C, Pyszkowska J. Obesity and pain. Hum NutrClin Nutr. 1983 Dec; 37C:307-310

4. Zahorska-Markiewicz B, Zych P, Kucio C. Pain sensitivity in obesity. Acta Physiol Pol. 1988 May-June; 39(3):183-187.

5. Khimich S. Level of sensitivity of pain in patients with obesity. Acta Chir Hung. 1997; 36(1-4):166-167.

6. McKendall MJ, Haier RJ. Pain sensitivity and obesity. Psychiatry Res. 1983 Feb; 8:119-125.

7. Karayiannakis AJ, Syrigos KN, Zbar A, Makri GG, Athanasiadis L, Alexiou $D$, et al. The effect of vertical banded gastroplasty on glucose-induced $\beta$-endorphin response. J Surg Res. 1998 Dec; 80:123-128.

8. Leknes S, Tracey I. A common neurobiology for pain and pleasure. Nature Rev Neurosci. 2008 Apr; 8:314-320.

9. Dayer JM, Chicheportiche R, Juge-Aubry C, Meier C. Adipose tissue has antiinflammatory properties. Ann NY Acad Sci. 2006 June; 1069:444-453.

10. Uceyler N, Eberle T, Rolke R, Birklein F, Sommer C. Differential expression patterns of cytokines in complex regional pain syndrome. Pain. 2007 Nov; 132(1-2):195-205

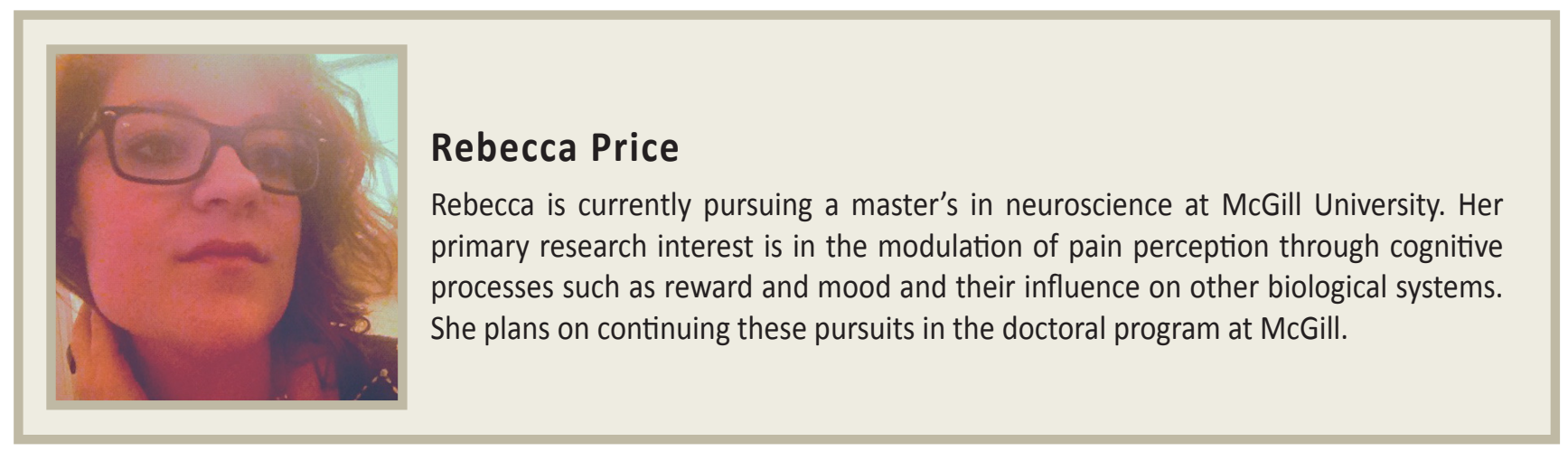

